\title{
Thermodynamically Guided Nonequilibrium Monte Carlo Method for Generating Realistic Shear Flows in Polymeric Systems
}

\author{
C. Baig and V. G. Mavrantzas* \\ Department of Chemical Engineering, University of Patras and FORTH-ICE/HT, Patras, GR 26504, Greece
}

(Received 20 June 2007; published 19 December 2007)

\begin{abstract}
A thermodynamically guided atomistic Monte Carlo methodology is presented for simulating systems beyond equilibrium by expanding the statistical ensemble to include a tensorial variable accounting for the overall structure of the system subjected to flow. For a given shear rate, the corresponding tensorial conjugate field is determined iteratively through independent nonequilibrium molecular dynamics simulations. Test simulations for the effect of flow on the conformation of a $\mathrm{C}_{50} \mathrm{H}_{102}$ polyethylene liquid show that the two methods (expanded Monte Carlo and nonequilibrium molecular dynamics) provide identical results.
\end{abstract}

PACS numbers: 61.20.Ja, 61.20.Gy, 61.25.Hq

Traditionally, the Monte Carlo (MC) method has been thought of as an efficient tool for simulating the properties of long-chain molecules only under equilibrium conditions, since it is a nontrivial matter to drive an ensemble of system configurations to sample statistically appropriate nonequilibrium phase-space points corresponding to an imposed external field. The first attempt to overcome such a limitation was made about 10 years ago [1] when a field-on MC procedure was designed and implemented to simulate chain molecules subjected to a homogeneous extensional flow at constant elongation rate in one direction (which has perhaps the simplest kinematical structure in nonequilibrium MC simulations of flowing systems). Although still quantitatively unrealistic, this first effort demonstrated the significant role of such a (synthetic) nonequilibrium thermodynamic force field in driving chain conformations. In a later study [2], it was shown how one can take advantage of the general equation for the nonequilibrium reversible-irreversible coupling (GENERIC) formalism of nonequilibrium thermodynamics, a widely applied thermodynamic framework of a number of physical systems [3]. It was shown how to formulate rigorously MC simulation schemes in well-defined expanded or generalized statistical ensembles. Key elements of the new simulation technique, also termed the GENERIC MC method, are the vector $\mathbf{x}$ of the proper state variables, the vector $\boldsymbol{\lambda}$ of the corresponding conjugate fields, and the relevant probability density function $\rho_{[\lambda]}$.

Despite its thermodynamic foundation, however, it has always remained an open question whether or not the GENERIC MC method is capable of generating or sampling the true nonequilibrium structure of the system under a given flow field. More specifically, the following questions remained open: Would such a formal extension of the equilibrium statistical thermodynamics (through the introduction of additional field terms) be justified even for systems far beyond equilibrium? If yes, would then the choice of the (coarse-grained) thermodynamic variables and of their conjugate force fields (the so-called
Lagrange multipliers in GENERIC) be good enough to reproduce such a complicated nonequilibrium structure of the system? And, more importantly, would it be possible to give such synthetic force terms a clear physical meaning in relation to the real flow fields? For example, can one establish an analytical correlation between the Lagrange multiplier and the velocity gradient tensor in shear and elongational flows?

We address many of these issues in this Letter by directly comparing the results of the GENERIC MC method with those obtained through a brute-force application of the nonequilibrium molecular dynamics (NEMD) method. The comparison is made for the case of shear flow for two reasons: (a) This is considered as one of the most difficult cases to generate with the GENERIC MC method because it requires a rather complicated form of the Lagrange multiplier, and (b) it is the most important flow with the widest applications in rheology.

In the generalized canonical GENERIC ensemble [3], the probability density function $\rho_{[\lambda]}$ is written as

$$
\begin{aligned}
\rho_{[\boldsymbol{\lambda}]}(\mathbf{z}) & =\frac{1}{Q(\boldsymbol{\lambda})} \exp \left(-\sum_{k} \lambda_{k} \Pi_{k}(\mathbf{z})\right) \\
Q(\boldsymbol{\lambda}) & =\int \exp \left(-\sum_{k} \lambda_{k} \Pi_{k}(\mathbf{z})\right) d \mathbf{z},
\end{aligned}
$$

where $\mathbf{z}$ denotes the phase space consisting of the atomistic position and momentum coordinates. The Lagrange multiplier $\lambda_{k}$ represents an intensive thermodynamic force exciting its conjugate extensive thermodynamic quantity, $\Pi_{k}$. The corresponding thermodynamic state variable $x_{k}$ is simply the average of $\Pi_{k}$. The total set of state variables $\mathbf{x}$ of the physical system is typically expressed as $\mathbf{x}=$ $\{\rho(\mathbf{r}), \mathbf{u}(\mathbf{r}), \varepsilon(\mathbf{r}), \mathbf{X}(\mathbf{r})\}$, where $\rho$ is the mass density, $\mathbf{u}$ the momentum density, $\varepsilon$ the internal energy density, $\mathbf{X}$ the structural variable(s) characterizing nonequilibrium states, and $\mathbf{r}$ the position vector. The Lagrange multipliers $\lambda_{k}$ are defined through 


$$
\frac{\delta S(\mathbf{x})}{\delta x_{k}}=k_{B} \lambda_{k}
$$

where $S$ denotes the entropy. Based on this definition and the fundamental evolution law of the GENERIC formalism for the set of state variables $\mathbf{x}$ [3], we find that, under steady-state conditions, the Lagrange multipliers obey the kinematic equation

$$
\lambda=-\frac{1}{k_{B}} \mathbf{M}^{-1} \cdot \mathbf{L}(\mathbf{x}) \cdot \frac{\delta E(\mathbf{x})}{\delta \mathbf{x}} .
$$

While $\mathbf{L}(\mathbf{x})$ and $E(\mathbf{x})$ in Eq. (3), controlling the Hamiltonian reversible dynamics of system, are relatively well known for many physical systems [3], the form of the friction matrix $\mathbf{M}(\mathbf{x})$ representing the irreversible dissipative dynamics depends on the physical system under consideration and usually requires physical insight to define.

We restrict ourselves here to the simplest but still most successful case of the coarse-grained structural variable $\mathbf{X}$ for a system under flow, namely, the conformation tensor $\tilde{\mathbf{c}}$ [3-5]. Then, following Mavrantzas and Theodorou [1], we extend the Helmholtz free energy $A$ of equilibrium systems to nonequilibrium systems as

$$
d\left(\frac{A}{V}\right)=-\frac{S}{V} d T+\mu d\left(\frac{N}{V}\right)+\frac{N}{V} k_{B} T \boldsymbol{\alpha}: d \tilde{\mathbf{c}},
$$

where $V$ is the volume of the system, $T$ the temperature, $N$ the number of molecules, $\mu$ the chemical potential, and $k_{B}$ Boltzmann's constant. The last term accommodates the effect of an external field for which $\boldsymbol{\alpha}$ represents a nonequilibrium force variable conjugate to $\tilde{\mathbf{c}}$. $\boldsymbol{\alpha}$ is directly related to the Lagrange multiplier $\boldsymbol{\lambda}$ in the GENERIC formalism $[\boldsymbol{\lambda}=-(N / V) \boldsymbol{\alpha}]$.

In the case of simple shear flow, from the symmetry property of $\tilde{\mathbf{c}}$, it is easily recognized that $\boldsymbol{\alpha}$ is to have only four independent nonzero components: $\alpha_{x x}, \alpha_{x y}, \alpha_{y y}$, and $\alpha_{z z}$. In order to find the numerical values of these four components for a given shear rate (to be used as input to the GENERIC MC simulations), one can resort to known viscoelastic models such as the upper-convected Maxwell, the Giesekus, the finitely extensible nonlinear elastic with the Cohen approximation (FENE-C), and the hybrid FENE-C/Giesekus model [4]. For all of them, the evolution equation can be expressed in the form of [4]

$$
\begin{aligned}
& \hat{\tilde{c}}_{\alpha \beta}=-\Lambda_{\alpha \beta \gamma \varepsilon} \frac{\delta A(\tilde{\mathbf{c}})}{\delta \tilde{c}_{\gamma \varepsilon}}=-n k_{B} T \Lambda_{\alpha \beta \gamma \varepsilon} \alpha_{\gamma \varepsilon} ; \\
& \alpha_{\alpha \beta}=\frac{1}{n k_{B} T} \frac{\delta A(\tilde{\mathbf{c}})}{\delta \tilde{c}_{\alpha \beta}} .
\end{aligned}
$$

Here $\hat{\tilde{c}}_{\alpha \beta}$ denotes the upper-convected derivative of $\tilde{c}_{\alpha \beta}$ and $n$ the chain number density, while the Einstein summation convention has been assumed over repeated indices. Note also that, in the case considered here, the element $M_{44}$ of the M matrix in Eq. (3) has the form of $T \Lambda_{\alpha \beta \gamma \varepsilon}$ [2] where the fourth-order relaxation matrix $\Lambda$ for most single- conformation tensor models can be cast into the following general form:

$$
\begin{aligned}
\Lambda_{\alpha \beta \gamma \varepsilon}(\tilde{\mathbf{c}})= & f_{1}\left(I_{1}\right)\left(\tilde{c}_{\alpha \gamma} \delta_{\beta \varepsilon}+\tilde{c}_{\alpha \varepsilon} \delta_{\beta \gamma}+\tilde{c}_{\beta \gamma} \delta_{\alpha \varepsilon}\right. \\
& \left.+\tilde{c}_{\beta \varepsilon} \delta_{\alpha \gamma}\right)+2 f_{2}\left(I_{1}\right)\left(\tilde{c}_{\alpha \gamma} \tilde{c}_{\beta \varepsilon}+\tilde{c}_{\alpha \varepsilon} \tilde{c}_{\beta \gamma}\right) .
\end{aligned}
$$

In Eq. (6), $I_{1}$ is the first invariant (the trace) of $\tilde{\mathbf{c}}, \boldsymbol{\delta}$ the unit tensor, and $f_{1}$ and $f_{2}$ arbitrary functions of $I_{1}$. When applying Eqs. (5) and (6) to the simple steady-state shear flow described by the kinematics

$$
\boldsymbol{\nabla} \mathbf{u}=\left(\begin{array}{ccc}
0 & 0 & 0 \\
\dot{\gamma} & 0 & 0 \\
0 & 0 & 0
\end{array}\right),
$$

$\alpha_{z z}$ turns out to be identically zero. The same reduction has been adopted in the current work in order to study its consequences and thus appreciate the suggestions of the known viscoelastic models. That is, we assume that the form of $\boldsymbol{\alpha}$ that generates a shear flow is

$$
\boldsymbol{\alpha}=\left(\begin{array}{ccc}
\alpha_{x x} & \alpha_{x y} & 0 \\
\alpha_{x y} & \alpha_{y y} & 0 \\
0 & 0 & 0
\end{array}\right) .
$$

With the form of Eq. (8) for $\boldsymbol{\alpha}$, GENERIC MC simulations were executed in the semigrand canonical ensemble [6] for a system consisting of 120 linear $\mathrm{C}_{50} \mathrm{H}_{102}$ molecules in a rectangular box, enlarged in the $x$ direction (flow direction), with dimensions $(x \times y \times z)$ of $93 \times 45 \times$ $45 \AA^{3}$ ( $y$ and $z$ axes are chosen as the velocity gradient and the neutral directions, respectively). We chose 5 different nonequilibrium states corresponding to 5 different values of the Deborah (De) number equal to 0.425, 1.06, 4.25, 21.3, and 106. De is defined as the product of the imposed shear rate $\dot{\gamma}$ and the longest relaxation time of the system, $\tau_{R}$; here, $\tau_{R} \approx 0.5 \mathrm{~ns}$ as estimated by the integral below the curve describing the time decay of the autocorrelation function for the chain end-to-end vector.

For each one of these 5 values of De, initial values for the 3 parameters $\alpha_{x x}, \alpha_{x y}$, and $\alpha_{y y}$ were obtained by solving a hybrid FENE-C/Giesekus model for the given De. With an initial value for $\boldsymbol{\alpha}$, GENERIC MC simulations were executed with the well-known connectivity-altering end-bridging move [6], allowing for a small polydispersity index $I(\approx 1.083)$ and a uniform distribution of chain lengths in the simulated system. All GENERIC MC simulations were performed with the SKS united-atom potential model [7], at temperature $T=450 \mathrm{~K}$ and mass density $\rho=0.744 \mathrm{~g} / \mathrm{cm}^{3}$. For any given De, the results of the GENERIC MC simulation for $\tilde{\mathbf{c}}$ at the corresponding value of the Lagrange multiplier $\boldsymbol{\alpha}$ were compared against those obtained by a brute-force execution of the NEMD method for the same De [8]. Although the initial values of $\boldsymbol{\alpha}$ suggested by the FENE-C/Giesekus model were very useful to start with, they turned out not to be sufficiently accurate. Thus, a few iterations (6-8) were required for 


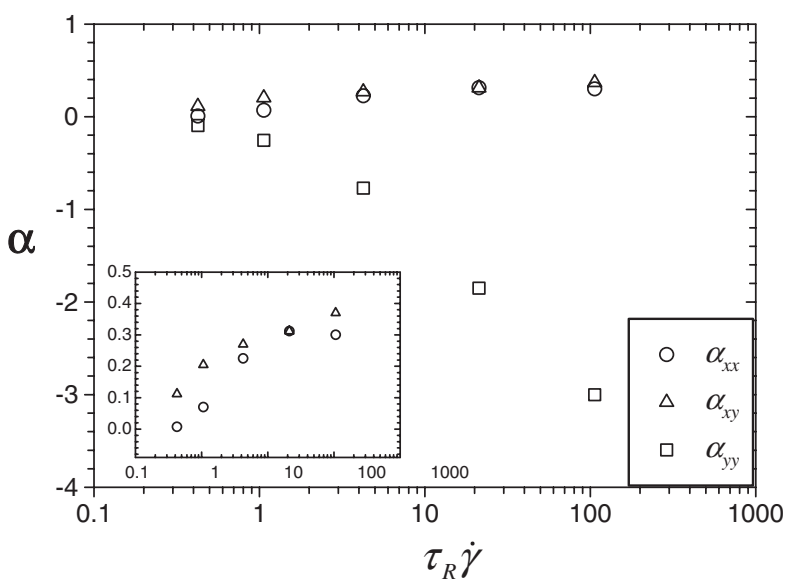

FIG. 1. Plot of the thermodynamic force field $\boldsymbol{\alpha}$ versus De.

each state point in order for GENERIC MC and NEMDbased simulation results to converge, for the given value of shear rate. The objective function minimized in each such iteration was essentially the root mean square of the deviation of the GENERIC MC data for $\tilde{\mathbf{c}}$ from the corresponding NEMD data:

$$
F_{\mathrm{obj}}=\sqrt{\frac{\sum_{i=1}^{n_{\mathrm{type}}} w_{i}\left[\left(R_{i, \mathrm{GENERIC} \mathrm{MC}}-R_{i, \mathrm{NEMD}}\right) / R_{i, \mathrm{NEMD}}\right]^{2}}{\sum_{i=1}^{n_{\mathrm{type}}} w_{i}}},
$$

where $n_{\text {type }}$ is the number of data types, $w_{i}$ a weighting factor, and $R$ the result for $\tilde{\mathbf{c}}$ either from the GENERIC MC method or from the NEMD simulation. Since in this work a zero value was chosen for $\alpha_{z z}$, the contribution of $\tilde{c}_{z z}$ was removed and $n_{\text {type }}$ was thus equal to $3\left(\tilde{c}_{x x}, \tilde{c}_{x y}\right.$, and $\left.\tilde{c}_{y y}\right)$. Also, in this work, the same weight was used for $\tilde{c}_{x x}, \tilde{c}_{x y}$, and $\tilde{c}_{y y}$; i.e., $w\left(\tilde{c}_{x y}\right)=w\left(\tilde{c}_{x x}\right)=w\left(\tilde{c}_{y y}\right)$.
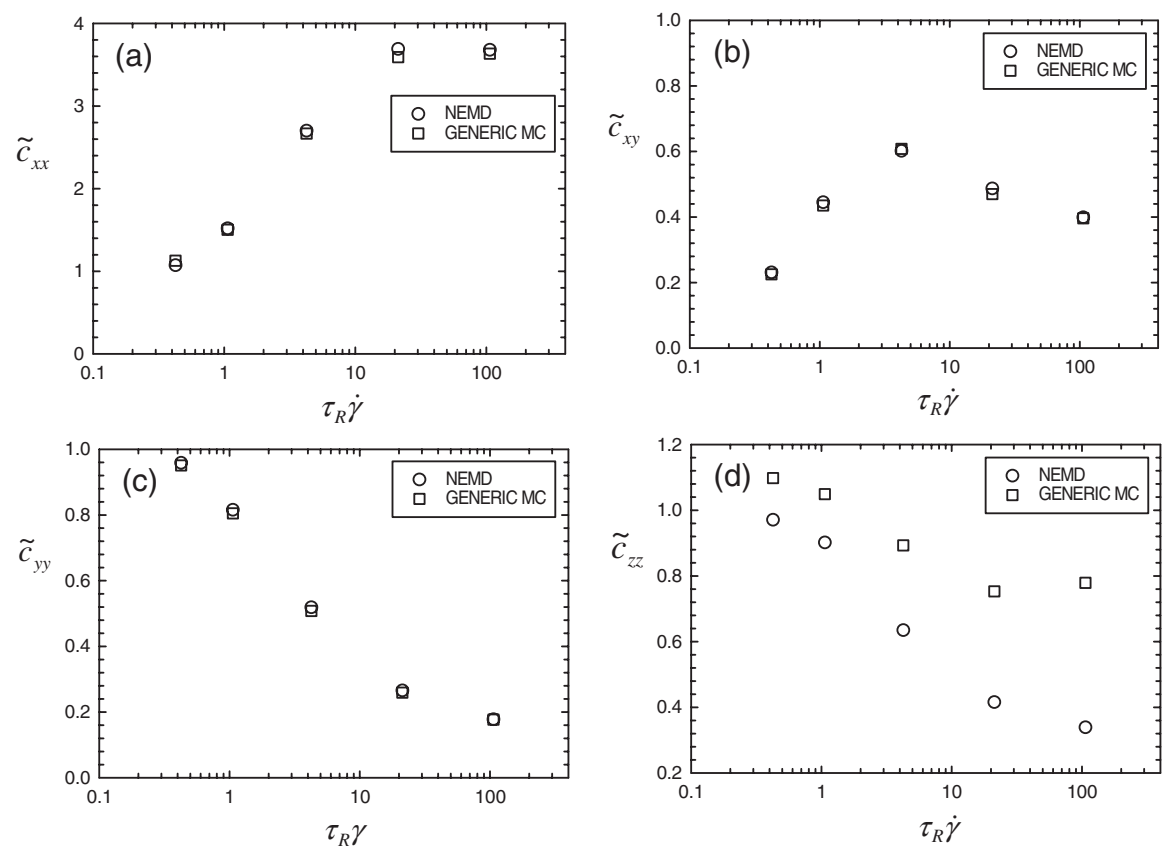

$257801-3$
Figure 1 presents the values of the 3 components of the tensor $\boldsymbol{\alpha}$ that were found to reproduce accurately the corresponding nonequilibrium state for the simulated $\mathrm{C}_{50} \mathrm{H}_{102}$ system at each shear rate, as a function of the imposed De. $\alpha_{x x}$ and $\alpha_{x y}$ appear to increase with increasing shear rate, a trend which is considered physically reasonable, since chains are to be more and more oriented and extended along the $x$ direction with increasing shear rate. From our experience with the iterative procedure followed here, an increase in the value of $\alpha_{x x}$ or $\alpha_{x y}$ usually results in an increase in the values of both $\tilde{c}_{x x}$ and $\tilde{c}_{x y}$. Furthermore, $\alpha_{x x}$ has a stronger effect on $\tilde{c}_{x x}$ than $\alpha_{x y} ; \alpha_{x y}$, on the other hand, is correlated with $\tilde{c}_{x y}$ and $\tilde{c}_{y y}$ more than $\alpha_{x x}$. On the contrary, $\tilde{c}_{z z}$ always appeared to decrease upon increasing either $\alpha_{x x}$ or $\alpha_{x y}$. For all strain rates, $\tilde{c}_{y y}$ was found to be well controlled by $\alpha_{y y}$; the latter was always negative and its magnitude was shown to increase rather fast compared to $\alpha_{x x}$ and $\alpha_{x y}$.

It is very important to note that the above information for the dependence of $\boldsymbol{\alpha}$ on De can be used to calculate the (nonequilibrium) free energy of system - see Eq. (4) through thermodynamic integration. This is extremely useful in the theoretical development of nonequilibrium thermodynamics, as well as in developing viscoelastic models.

Comparisons were made of the conformation tensor components between the GENERIC MC simulations (corresponding to the $\boldsymbol{\alpha}$ values shown in Fig. 1) and the direct NEMD simulations. As seen in Figs. 2(a)-2(c), $\tilde{c}_{x x}, \tilde{c}_{x y}$, and $\tilde{c}_{y y}$ from the GENERIC MC method are all in excellent agreement with their corresponding values from NEMD simulations at each shear rate studied. The same was found to be true for the birefringence, especially for De numbers less than 10. These results confirm that our thermodynamically guided GENERIC MC methodology is capable of
FIG. 2. Comparison of the conformation tensor components between NEMD and GENERIC MC simulations, as a function of De number: (a) $\tilde{c}_{x x}$, (b) $\tilde{c}_{x y}$, (c) $\tilde{c}_{y y}$, and (d) $\tilde{c}_{z z}$. The error bars are smaller than the size of the symbols. 


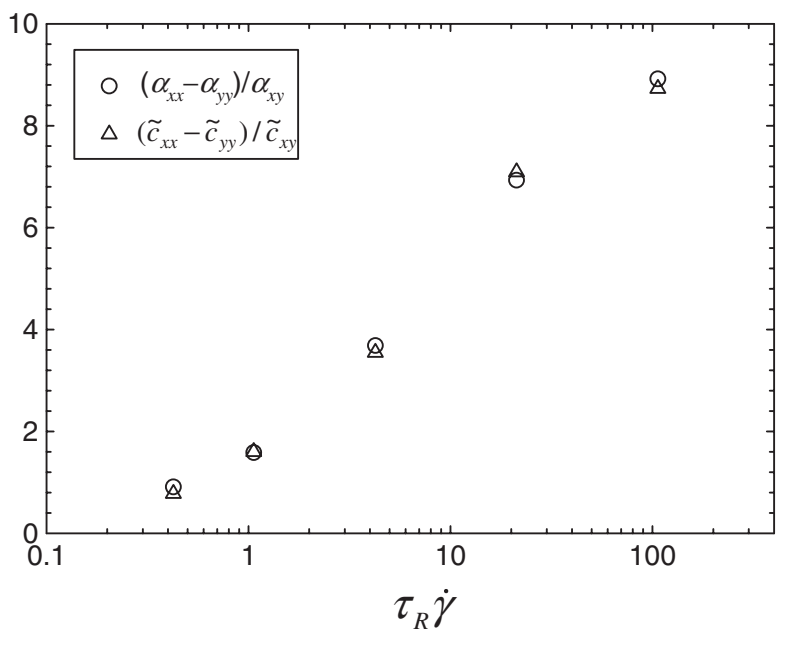

FIG. 3. Comparison between $\left(\alpha_{x x}-\alpha_{y y}\right) / \alpha_{x y}$ and $\left(\tilde{c}_{x x}-\right.$ $\left.\tilde{c}_{y y}\right) / \tilde{c}_{x y}$ at each shear rate.

correctly reproducing a nonequilibrium structure similar to that observed in the real system under flow. It is only for the $\tilde{c}_{z z}$ component of the conformation tensor $\tilde{\mathbf{c}}$ that the agreement between MC and NEMD results is relatively poor. This is related to our selection of a zero value for the $\alpha_{z z}$ component of $\boldsymbol{\alpha}$, as suggested by the currently available conformation tensor models, which apparently is not an appropriate choice. The prediction for $\tilde{c}_{z z}$ would not be improved even if one made use of a linear superposition of multiple conformation tensors as long as a zero value of $\alpha_{z z}$ is chosen for each individual mode. Only by allowing for couplings between the different modes, $\tilde{c}_{z z}$ might be improved to some extent; however, such a coupling cannot be justified on firm physical basis. This argument is also in line with the short length of the simulated polyethylene system $\left(\mathrm{C}_{50} \mathrm{H}_{102}\right)$ for which higher modes are less important compared to the first one. Clearly, to reproduce exactly $\tilde{c}_{z z}$, a nonzero $\alpha_{z z}$ component should be incorporated in the GENERIC MC method. Our simulations, therefore, suggest that the rather general form of the friction matrix, Eq. (6), characterizing this family of models needs to be improved. This is quite important since the $a_{z z}$ component is directly related with the capability of the model(s) to reproduce the second normal stress coefficient.

Based on the GENERIC [3] and/or generalized bracket [4] expressions for the elastic part of the stress tensor, simple symmetry requirements lead also to the following important relation between $\boldsymbol{\alpha}$ and $\tilde{\mathbf{c}}$ in shear: $\frac{\alpha_{x x}-\alpha_{y y}}{\alpha_{x y}}=$ $\frac{\tilde{c}_{x x}-\tilde{c}_{y y}}{\tilde{c}_{x y}}$. The GENERIC MC results are shown in Fig. 3, and they nicely confirm that the employed methodology indeed generates the correct structure of the stress tensor in the $\mathrm{C}_{50} \mathrm{H}_{102}$ system.

In conclusion, we have presented the first successful effort to use a nondynamic method in order to generate a realistic shear flow of an unentangled polymer liquid. In addition to providing valuable information for the true forms of free energy functions for systems beyond equi- librium, the present work has other beneficial aspects. For example, it would be feasible to combine GENERIC MC simulations with NEMD simulations in order to calculate faster the important dynamic properties of the melt. Also, combining the proposed GENERIC MC methodology with useful coarse-grained models that have appeared recently in the literature at different levels of coarse graining [9] could make it possible to investigate the viscoelastic properties of truly long-chain polymers, comparable to those encountered in practical polymer processing.

On the other hand, and despite the success of the proposed methodology, still a number of issues remain. For instance, while the GENERIC MC method can guide us in the development of a more accurate viscoelastic model, a good model would in turn facilitate the GENERIC MC method by offering more accurate initial guesses for $\boldsymbol{\alpha}$ for a given shear rate. In the meantime, it would also be worthwhile to put effort into clarifying if there exists a general relation between $\boldsymbol{\alpha}$ and strain rate under not only shear but also other types of flow, taking again into account the effect of chain length. This can be achieved only if all three different methodologies (GENERIC MC, NEMD, and macroscopic viscoelastic modeling) come together to complement each other while simultaneously avoiding empiricism.

We are grateful to Dow Benelux B. V. (especially to Dr. Joey Storer, Dr. Jaap den Doelder, and Dr. Rudy Koopmans) for financial support in the course of this project, but above all for their genuine interest and enthusiasm in this work. Enlightening discussions with Professor Hans Christian Öttinger and Professor Brian Edwards are deeply acknowledged.

*Author to whom correspondence should be addressed. vlasis@chemeng.upatras.gr

[1] V. G. Mavrantzas and D. N. Theodorou, Macromolecules 31, 6310 (1998).

[2] V. G. Mavrantzas and H. C. Öttinger, Macromolecules 35, 960 (2002)

[3] H.C. Öttinger, Beyond Equilibrium Thermodynamics (John Wiley \& Sons, New Jersey, 2005).

[4] A. N. Beris and B. J. Edwards, Thermodynamics of Flowing Systems (Oxford University Press, New York, 1994).

[5] R. B. Bird et al., Dynamics of Polymeric Liquids, Kinetic Theory (Wiley-Interscience, New York, 1987), 2nd ed., Vol. 2.

[6] P. V. K. Pant and D. N. Theodorou, Macromolecules 28, 7224 (1995); V. G. Mavrantzas et al., Macromolecules 32, 5072 (1999).

[7] J. I. Siepmann, S. Karaborni, and B. Smit, Nature (London) 365, 330 (1993).

[8] C. Baig et al., Rheol. Acta (to be published).

[9] R. Everaers et al., Science 303, 823 (2004); M. Kröger, Comput. Phys. Commun. 168, 209 (2005); C. Tzoumanekas and D. N. Theodorou, Macromolecules 39, 4592 (2006); V. A. Harmandaris et al., Macromolecules 39, 6708 ( 2006). 